\title{
Scale invariances and Lamperti transformations for stochastic processes
}

\author{
Pierre Borgnat $^{1}$, Pierre-Olivier Amblard ${ }^{2}$ and Patrick Flandrin ${ }^{1}$ \\ ${ }^{1}$ Laboratoire de Physique (UMR CNRS 5672) ENS Lyon, 46 allée d'Italie, \\ 69364 Lyon Cedex 07, France \\ 2 Laboratoire des Images et Signaux (UMR CNRS 5083) ENSIEG, BP 46, 38402 \\ Saint-Martin-d'Hères Cedex, France \\ E-mail: Pierre.Borgnat@ens-lyon.fr
}

Received 20 October 2004, in final form 30 December 2004

Published 23 February 2005

Online at stacks.iop.org/JPhysA/38/2081

\begin{abstract}
Scale-invariant processes, and hereafter processes with broken versions of this symmetry, are studied by means of the Lamperti transformation, a one-toone transformation linking stationary and self-similar processes. A general overview of the use of the transformation, and of the stationary generators it builds, is given for modelling and analysis of scale invariance. We put an emphasis on generalizations to non-strictly scale-invariant situations. The examples of discrete scale invariance and finite-size scale invariance are developed by means of the Lamperti transformation framework, and some specific examples of processes with these generalized symmetries are given.
\end{abstract}

PACS numbers: 02.50.Ey, 89.75.Da, 05.40.-a

\section{Lamperti transformation: a new reading}

\subsection{Scale invariance and beyond}

Scale invariance, once acknowledged as an important feature [39], has often been used as a fundamental property to handle physical phenomena. The idea that some quantity behaves the same at each scale, irrelevant to the scale at which it is observed, has made its way into the study of geometrical fractal sets [21], $1 / f$ spectra, long memory [6], simple dimensional analysis [5] or involved analysis of critical systems in statistical physics [22], textures in geophysics [52] or image processing [38], turbulence of fluids [27], data of network traffic [45] and so on.

Common as this invariance may be in physics, it often eludes general and convenient methods or models. Even though there is no single definition of scale invariance [19], it is often described as a symmetry of the system relatively to a transformation of scale, that is 
mainly a dilation or a contraction (up to some renormalization) of the system parameters. A first problem is that this symmetry is not always compatible with the usual symmetries such as stationarity in space or time, or isotropy, short-term memories and so on. In those cases, the meaning of the Fourier spectrum or correlation functions may be unclear or improper, and simple models such as Langevin equations or auto-regressive systems are not sufficient. Another issue is that, as with all symmetries, one expects real systems to experience symmetry breaking: incomplete invariance under dilations because of some additive part when scale is changed (self-affinity in fractals [21], renormalization equation of free energy in critical systems [22]), or the invariance holds only for some part of the meaningful scales (and sometimes infinite zooming is unmeaningful) [17, 43], else there exists some preferred scale ratios and the invariance stands true only for those scale ratios (the so-called discrete scaleinvariance property) [51].

We propose here to give a fresh look at the methodology for scale invariances (exact or incomplete) of stochastic processes; instead of dealing directly with the scale-invariant signal, one may transform the signal in some image that has a better known invariance such as stationarity. This approach comes from a generalization of the Lamperti theorem [35] which relates stationarity and self-similarity, and it is close to the extension of the concept of what stationarity is, as proposed by Hannan [33]: one can study invariances such as stationarity or self-similarity in similar frameworks. We argue hereafter that there exist such stationarizing transformations for exact or broken scale invariances and that effective methods for modelling and analysing scale invariances can be derived from this. We advocate the use of such a transform to define and study scale invariance, exact invariance or its many variations as broken symmetries.

This paper is then organized to cover two aspects of the Lamperti transformation: its usefulness for self-similarity and new insights about its generalization for broken scale invariance. By means of the Lamperti transform, we provide a new way of handling self-similar processes that leads to some methods of synthesis and analysis of exact selfsimilarity. The stationarization of self-similar processes was studied piecewise for specific applications by different authors and we give here a general formulation of the method, with new comments on its numerical applicability. This is the scope of the current section. The idea of stationarization is generalized in section 2 to encompass various forms of broken scale invariance. The specific case of discrete scale invariance [51] is studied in section 3 . The corresponding stochastic property is defined and elaborated here by means of the Lamperti transformation. Then a fourth section is devoted to broken scale invariances defined by a distortion of the dilation operators; this will provide insights into generalized scaling laws, especially the property of finite-size scale invariance [17] in the framework of the Lamperti transformation.

\subsection{Definition and property of the Lamperti transformation}

The roots of this work is the paper of Lamperti on scale invariance for stochastic processes [35], where he first pointed out a one-to-one correspondence between scale-invariant processes and stationary processes. To define what a dilation is, and thus the precise meaning of scale invariance, we use here the framework of stochastic representation of signals (for instance fluctuations or noises).

The following is the proper formalism for stochastic processes [20]. A random process $\{X(t), t>0\}$ is said to be self-similar of index $H$ (or scale invariant, denoted ' $H$-ss') if for any $\lambda \in \mathbb{R}_{*}^{+}$,

$$
\left\{\left(\mathcal{D}_{H, \lambda} X\right)(t) \hat{=} \lambda^{-H} X(\lambda t), t>0\right\} \stackrel{d}{=}\{X(t), t>0\}
$$


where $\stackrel{d}{=}$ stands for the 'equality' of the stochastic processes, that is equality of all joint finitedimensional distributions. This symmetry is an invariance under any renormalized dilation $\mathcal{D}_{H, \lambda}$ by a scale factor $\lambda, \mathcal{D}_{H, \lambda}$ being defined in the preceding equation. The theorem introduced by Lamperti in 1962 [35] uses the invertible transformation $\mathcal{L}_{H}{ }^{-1}$, acting on $\{X(t), t>0\}$ :

$$
\left(\mathcal{L}_{H}{ }^{-1} X\right)(t) \hat{=} \mathrm{e}^{-H t} X\left(\mathrm{e}^{t}\right)=Y(t), \quad t \in \mathbb{R} .
$$

Considering $\mathcal{L}_{H}{ }^{-1}$ as an inverse transformation, the corresponding direct transformation $\mathcal{L}_{H}$ is called the Lamperti transformation and is given by

$$
\left(\mathcal{L}_{H} Y\right)(t) \hat{=} t^{H} Y(\log t)=X(t), \quad t>0 .
$$

The theorem states that a process $\{X(t), t>0\}$ is $H$-ss if and only if $\{Y(t)=$ $\left.\left(\mathcal{L}_{H}{ }^{-1} X\right)(t), t \in \mathbb{R}\right\}$ is stationary; we call $Y(t)$ the stationary generator of the process $X(t)$. We recall that stationarity is the invariance under any time shift (or space shift if the parameter is space). For any $\tau \in \mathbb{R}$, a process $\left\{Y(t)=\left(\mathcal{L}_{H}{ }^{-1} X\right)(t), t \in \mathbb{R}\right\}$ has thus to satisfy $\left(\mathcal{S}_{\tau} Y\right)(t) \hat{=} Y(t+\tau) \stackrel{d}{=} Y(t)$. The core of the theorem is a mapping of operators such that for any $\lambda \in \mathbb{R}_{*}^{+}$, the Lamperti transformation guarantees that

$$
\mathcal{L}_{H}{ }^{-1} \mathcal{D}_{H, \lambda} \mathcal{L}_{H}=\mathcal{S}_{\log \lambda}
$$

Quoted in review books or articles (e.g., $[16,53,48,20]$ ), the theorem is completed by the unicity result of the transformation mapping stationarity to self-similarity, demonstrated by Burnecki et al [13].

\subsection{Properties of stationary generators of $H$-ss processes}

There have been few uses of the result of Lamperti beside mathematical works on selfsimilarity. Gray and Zhang [32] or Yazici and Kashyap [57] summoned some form of Lamperti's theorem to study specific classes of self-similar processes; Nuzman and Poor [44] and more recently Lim and Muniandy [36] used it extensively for fractional Brownian motions only. But those are among the few practical uses of the transformation. We have introduced general results on this subject in $[11,26]$, and we point out here their practical consequences for the modelling and analysis of scale invariance.

1.3.1. Covariance and spectrum of $H$-ss processes. The covariance of a scale-invariant random process $\{X(t), t>0\}$ admits necessarily the general form

$$
R_{X}(t, s) \hat{=} \mathbb{E}\{X(t) X(s)\}=(s t)^{H} C_{X}(t / s),
$$

where $C_{X}(e)$ is some non-negative definite function. In this expression, we have written $\mathbb{E}$ for the probabilistic expectation. The property (5) comes from the stationary generator $\left(Y=\mathcal{L}_{H}{ }^{-1} X\right)$ correlation function. The transformation assures that $R_{X}(t, s)=$ $(s t)^{H} R_{Y}(\log t, \log s)$. Hence, because of the stationarity of $Y$, its covariance depends only on the time difference, $R_{Y}(u, v)=c_{Y}(u-v)$. The central identity is therefore given for the correlation function $c_{Y}(\tau)=C_{X}\left(\mathrm{e}^{\tau}\right)$.

Our first and well-known example is the Brownian motion $B(t)$ defined as an integral of independent stationary Gaussian increments. $B(t)$ is also self-similar of index $1 / 2$ and thus it has a stationary generator. Its covariance is $R_{B}(t, s)=\sigma^{2} \max (t, s), \sigma^{2}$ being its variance. A simple calculus of the covariance of $\left(\mathcal{L}_{H}{ }^{-1} B\right)(t)$ gives $c_{\mathcal{L}_{H}{ }^{-1} B}(\tau)=\sigma^{2} \mathrm{e}^{-|\tau| / 2}$ and proves that this generator is the Ornstein-Uhlenbeck (OU) process, solution of the stationary linear Langevin equation driven by white noise. Consequently, the properties of the Brownian motion derive from the properties of the OU process $[16,20]$. 
The power spectrum $\Gamma_{Y}(f)$ of $Y(t)$ is the Fourier transform of the correlation function of a stationary process: $\boldsymbol{\Gamma}_{Y}(f)=\left(\mathbf{F} c_{Y}\right)(f)$. The Fourier transform is known as a suited representation for stationarity, but not for self-similarity. Starting from an $H$-ss process, the following algebra expresses the power spectrum of the stationary generator of a self-similar process:

$\left(\mathbf{F} c_{\mathcal{L}_{H}{ }^{-1} X}\right)(f)=\int_{-\infty}^{+\infty} C_{X}\left(\mathrm{e}^{\tau}\right) \mathrm{e}^{-\mathrm{i} 2 \pi f \tau} \mathrm{d} \tau=\int_{0}^{\infty} C_{X}(u) u^{-\mathrm{i} 2 \pi f-1} \mathrm{~d} u=\left(\mathbf{M} C_{X}\right)(\mathrm{i} 2 \pi f)$,

where $\mathbf{M}$ stands for the Mellin transform of the function. The definition of $\mathbf{M}$, for any function $g(u)$ and any variable $s \in \mathbb{C}$, is $(\mathbf{M} g)(s)=\int_{0}^{\infty} g(u) u^{-s-1} \mathrm{~d} u$.

The Mellin transform plays the same central role for self-similarity as the Fourier transform plays for stationarity because of the relation established in (6), which indeed is general for any quantity $g(u)$, given some index $H$ :

$$
(\mathbf{M} g)(H+\mathrm{i} 2 \pi f)=\int_{0}^{\infty} g(u) u^{-\mathrm{i} 2 \pi f-H-1} \mathrm{~d} u=\left(\mathbf{F} \mathcal{L}_{H}{ }^{-1} g\right)(f) .
$$

The basis functions are the Mellin chirps $\left\{t^{H+\mathrm{i} 2 \pi f}, t>0\right\}$, with $f \in \mathbb{R}$. Using this equivalence, note that one can obtain a harmonic-like representation of a self-similar process $X(t)$ as an inverse Mellin transform, namely an integral of uncorrelated spectral increments $\mathrm{d} \xi_{X}(f)$ on the Mellin basis [10, 26]:

$$
X(t)=\int_{0}^{\infty} t^{\mathrm{i} 2 \pi f+H} \mathrm{~d} \xi_{X}(f) .
$$

This is the Cramér representation for self-similar processes, mapped by $\mathcal{L}_{H}$ from the usual result known for stationary processes. It is valid under the assumption of the Loève condition, that is the summability of the integral representation for the second-order statistics of the process [37].

Here is obtained a spectral representation of an $H$-ss process with no assumption on stationarity (which is not compatible with self-similarity), nor on stationarity of the increments. With the added hypothesis of stationarity of the increments of the process, defined for any $\tau$ as $\{X(t+\tau)-X(t), t \in \mathbb{R}\}$, a good method to model the process is to use the wavelet transform [15]. It was proved really suited to study self-similar processes with stationary increments [1]. But, lacking this property, one cannot use the wavelet transform properly because there will not be convenient decorrelation between the coefficients. Also, because the wavelet transform with a wavelet $\psi$ reads as $T(a, t)=\int \psi(u) X(t-a u) \mathrm{d} u$, the scale $a$ defined with the wavelet transform is mainly based on the difference between two times. This is revealed by the underlying affine structure of the wavelet transform or by taking a look at one of the crudest wavelets: $\psi(u)=\left(\delta(u)-\delta\left(u-\tau_{0}\right)\right) / 2$. In this case, the wavelet transform is $T(a, t)=\left(X(t)-X\left(t-a \tau_{0}\right)\right) / 2$ (an expression close to the increment of the process) and the scale $a$ is probed by the difference of two times: $t_{1}=t-a \tau_{0}$ and $t_{2}=t$.

In contrast, the variable $f$ defined in (8) which is also a scale, named the Mellin scale (see for instance [24], p 210), is built on a ratio of times. It gives a simple description of how a process has changed between two times $t_{1}$ and $t_{2}$ : each component with Mellin scale $f$ has been multiplied by $\left(t_{2} / t_{1}\right)^{H+\mathrm{i} 2 \pi f}$. As dilations are defined by changing $t$ in $\lambda t$, the decomposition in Mellin scale is well suited to probe the behaviour under those dilations by the importance given to the ratio of two times, $t_{2} / t_{1}=\lambda$. The Mellin scale $f$ is the spectral variable associated with the dilation ratio, in the same sense that a Fourier frequency allows the effects of a time-shift to easily described. Thus, this Mellin scale is adapted to self-similarity where invariant properties under dilations are expected. Moreover, a spectral decomposition on Mellin scales will be an interesting tool for processing of self-similar processes. 
1.3.2. Scale-invariant filters and models. A further consequence is that scale-invariant linear systems are found as images by $\mathcal{L}_{H}$ of stationary (linear) filters. Applying $\mathcal{L}_{H}$ on a stationary filter $\mathcal{H}$ that has the usual action on $Y(t)$ as a convolution: $(\mathcal{H} Y)(t)=\int_{-\infty}^{+\infty} h(t-u) Y(u) \mathrm{d} u$, one defines systems having the form of a multiplicative convolution,

$$
(\mathcal{G} X)(t)=\int_{0}^{\infty} g(t / s) X(s) \frac{\mathrm{d} s}{s}=\int_{0}^{\infty} g(s) X(t / s) \frac{\mathrm{d} s}{s} .
$$

They are related to usual filters by means of $\mathcal{L}_{H}$ because we obtain this equation by setting $\left(\mathcal{L}_{H} h\right)(s)=g(s)$. Note that it is not a wavelet transform because it says nothing about time shifts here (we only have one variable). We put together the defining property of stationary filters which is that they commute with any time shift $\mathcal{S}_{\tau}$ (i.e., for any $\tau \in \mathbb{R}, \mathcal{H} \mathcal{S}_{\tau}=\mathcal{S}_{\tau} \mathcal{H}$ ) and the equivalence (4). Then one finds that a defining property of the scale-invariant filters $\mathcal{G}$ is that they commute with dilations (covariance with dilations), i.e., $\mathcal{G D}_{H, \lambda}=\mathcal{D}_{H, \lambda} \mathcal{G}$ for any $\lambda \in \mathbb{R}_{*}^{+}$. As such they preserve self-similarity and are a good tool to process self-similar signals without disturbing this key property.

Taking the Mellin transform of (9) one formally obtains a transfer function for those systems: $\mathrm{d} \xi_{\mathcal{G} X}(f)=(\mathbf{M} g)(\mathrm{i} 2 \pi f) \mathrm{d} \xi_{X}(f)$. From this, it is possible to design parametric models built on scale-invariant filters, by taking a rational function for the transfer function $(\mathbf{M} g)(s)$. The models are mapped by $\mathcal{L}_{H}$ from the ARMA models, and are found to follow Euler-Cauchy systems. A theory of parametric modelling for self-similarity may be written on these premises [11] and some issues were covered in [26] and in previous works [12, 56, 57] that did not use the Lamperti correspondence explicitly.

1.3.3. Fractional Brownian motions. From the above results, one can study a typical model of $H$-ss stochastic processes: the fractional Brownian motion $(\mathrm{fBm})\left\{B_{H}(t), t>0\right\}$. The fBm is the only (up to a multiplicative constant) Gaussian process that is $H$-ss, and has stationary increments [20,41]. When $H=1 / 2$, the fBm collapses onto the usual Brownian motion, the increments being therefore independent in that case. Its covariance has the form

$$
\begin{aligned}
R_{B_{H}}(t, s) & =\frac{\sigma^{2}}{2}\left(|t|^{2 H}+|s|^{2 H}+|t-s|^{2 H}\right) \\
& =\frac{\sigma^{2}}{2}|s t|^{H}\left(|t / s|^{H}+|s / t|^{H}+|\sqrt{t / s}-\sqrt{s / t}|^{2 H}\right),
\end{aligned}
$$

which has the expected form of (5). Using the inverse Lamperti transform, the stationary generator $\left\{Y_{H}(t), t \in \mathbb{R}\right\}$ of the fBm is shown to be a stationary Gaussian process with zero mean and covariance function given by $[26,36,44]$

$$
R_{Y_{H}}(\tau)=\frac{\sigma^{2}}{2}\left[\cosh (H \tau)-(2 \sinh (|\tau| / 2))^{2 H} / 2\right]
$$

When $H=1 / 2$, we recognize the covariance function of the Ornstein-Uhlenbeck process, else we obtain some kind of generalized OU process. For the point of view adopted here, the $\mathrm{fBm}$ has the following Mellin spectrum (or Fourier spectrum of its associated generator):

$$
\left(\mathbf{M} C_{B_{H}}\right)(\mathrm{i} 2 \pi f)=\Gamma_{Y_{H}}(f)=\frac{\sigma^{2}}{H^{2}+4 \pi^{2} f^{2}}\left|\frac{\Gamma(1 / 2+\mathrm{i} 2 \pi f)}{\Gamma(H+\mathrm{i} 2 \pi f)}\right|^{2},
$$

where $\Gamma$ is the Euler gamma function. Characterizations of the generalized OU process can be found in $[26,36,44]$. 

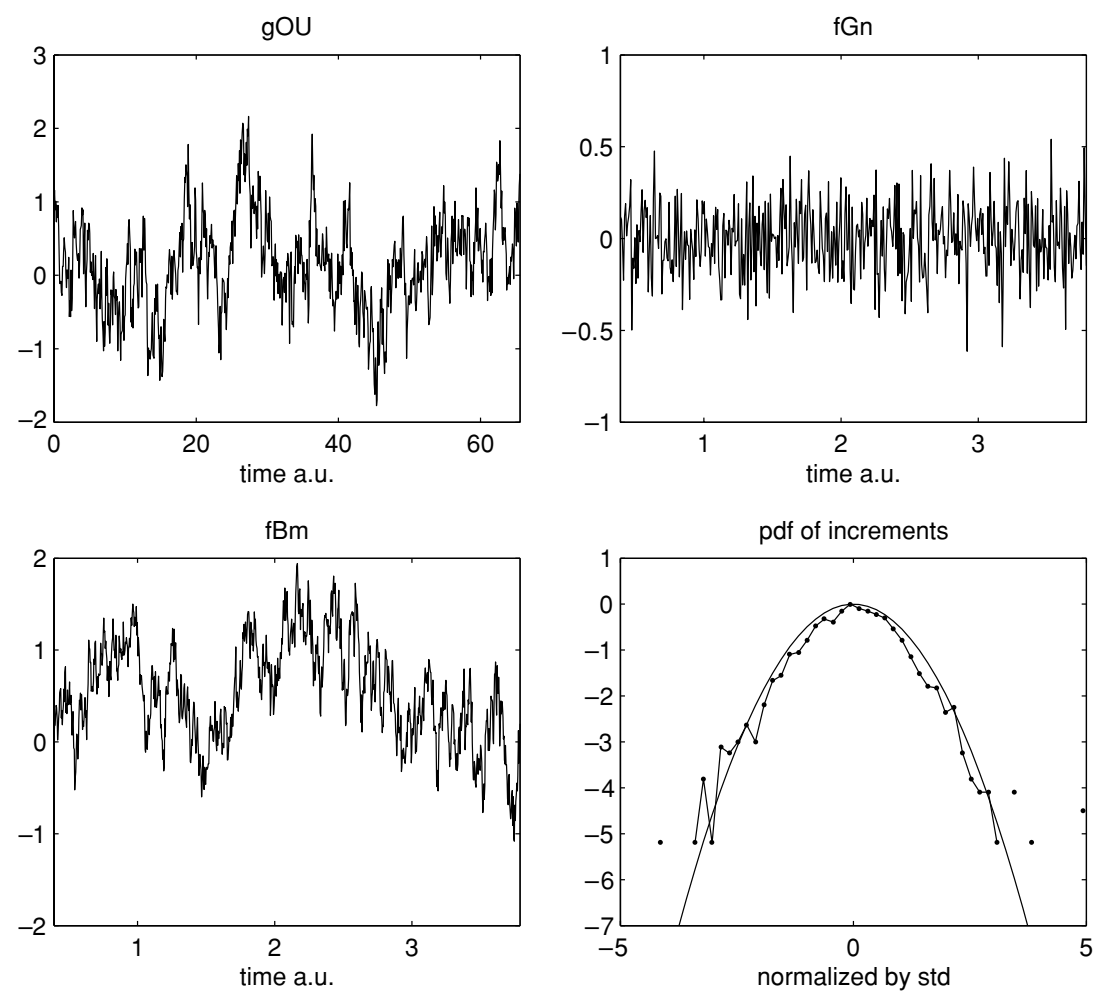

Figure 1. Example of fractional Brownian motion, with $H=0.3$, computed by means of its stationary generator. Top left: the generator $Y(t)$, synthetized from its stationary correlation function, (equation (12)) (using the method of the circulant matrix). Bottom left: the fBm deduced from the preceding process, by $B(t)=\left(\mathcal{L}_{H} Y\right)(t)$. Top right: the increments $B(t+1)-B(t)$ of the $\mathrm{fBm}$, known to be stationary. Bottom right: the logarithm of the histogram of the increments of the $\mathrm{fBm}$ that shows they are Gaussian as expected (the curve is the Gaussian on this lin-log scale).

\subsection{Use of Lamperti transform for numerical operations}

The Lamperti transform is twofold: a multiplication by a non-stationary factor $t^{H}$ and a change of the way the time variable is measured, from $t$ to $\log t$. The first part captures the general growth of the statistics driven by the $H$ exponent. The second part is a kind of clock change in order to find the natural scale for measuring the evolution of the process (which here is $\log t$ ), and is referred as a warping of the time in signal processing [4].

It is then possible to use this feature for numerical analysis or synthesis of discrete-time sequences. Because of the stationarity of the generator, a good way of synthesis for self-similar processes is to compute a realization of the generator by means of fast algorithms requiring stationarity (e.g., Levinson algorithm or the circulant matrix of Wood and Chan [55]). The generator is obtained as a uniformally sampled sequence $Y(t=n \tau)$ with a fixed $\tau$ and the indices $n$ in a subset of $\mathbb{Z}$. The transformed process is then known at times $t=\mathrm{e}^{n \tau}=\left(\mathrm{e}^{\tau}\right)^{n}$, thus with a geometrical sampling. By resampling the process, one can obtain a realization of the $H$-ss process with a uniform sampling. Figure 1 shows a typical snapshot of an $\mathrm{fBm}$ obtained this way and its associated generalized OU generator. Figure 2 illustrates that this method may also be used for models with no stationary increments (whereas an fBm has stationary increments), and therefore in situations where a fast algorithm is lacking. 

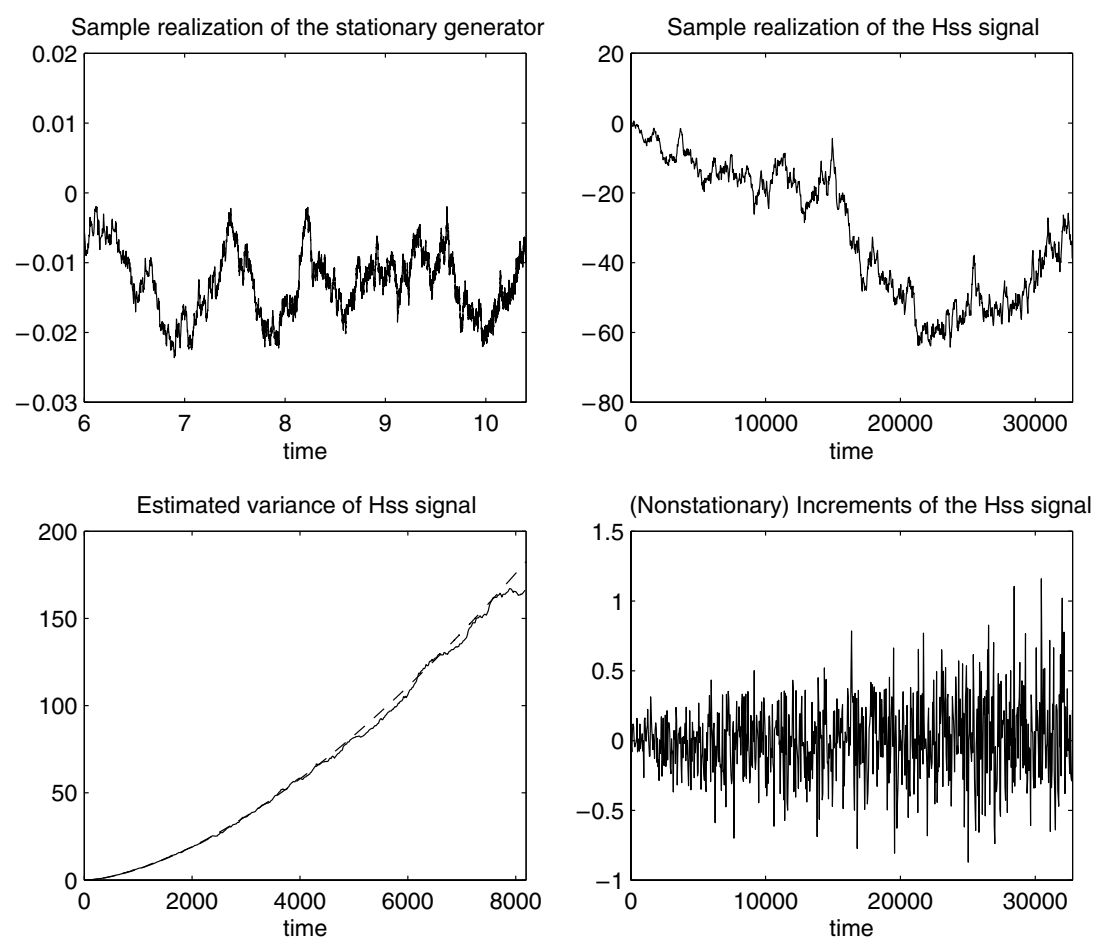

Figure 2. Example of a self-similar process, with $H=0.8$, computed by means of its stationary generator having covariance $c_{Y}(\tau)=\exp (-\alpha|\tau|)$. Top left: the generator $Y(t)$, synthesized from its stationary correlation function. Top right: the $H$-ss process deduced from the preceding process, implementing $X(t)=\left(\mathcal{L}_{H} Y\right)(t)$. Bottom left: estimated variance of the process, $\mathbb{E} X(t)^{2}$, done empirically on 1024 realizations; the expected curve in $\sigma^{2} t^{2 H}$ is drawn by a dashed line. Bottom right: the increments $X(t+1)-X(t)$, which are not stationary (so cannot be used to synthesize the process conveniently). This process is also the output of an Euler-Cauchy system of order 1, and has been studied in $[42,26]$.

For the sake of analysis, in figure 3 we illustrate that the Mellin spectrum may be more meaningful than a Fourier spectrum for an unknown self-similar process. On the one hand, the Fourier spectrum hardly says a thing where there is no stationarity, and is usually broadband. For self-similar processes, with an added assumption of stationary increments, one may deduce the $H$ exponent of the Fourier spectrum [6]. We indeed expect that it behaves as $f^{-2 H-1}$ over a large range of frequencies, but no other features are easy to understand with regard to self-similarity. On the other hand, one can expect $H$-ss processes to have a Mellin spectrum that could be modelled by Euler-Cauchy systems (in the same way that stationary processes are well modelled by auto-regressive systems), involving a reduced number of parameters. A numerical inverse Lamperti transform may be used in practical implementation [11]. A central argument is the possibility of using a fast Mellin transform algorithm (based on FFT), as was studied in [8]. The exponent $H$ is an external parameter of the transform and should be given a priori, estimated from a Fourier spectrum, or found by trying several exponents $H$ until one matches. Another argument in favour of the practical use of the Lamperti transform is the not so great sensitivity of the spectral analysis to the choice of the renormalization index $H$ : being mistaken in the choice of $H$ broadens a little the peaks in the Mellin transform. For instance, the numerical transform of $t^{H+\mathrm{i} 2 \pi f_{0}}$ would not be the Dirac mass in $f_{0}$ but a Lorenzian function of width $\sqrt{3}|H-r| /(\pi T)$ at half-height if the renormalization exponent is $r$ instead of $H$, and 

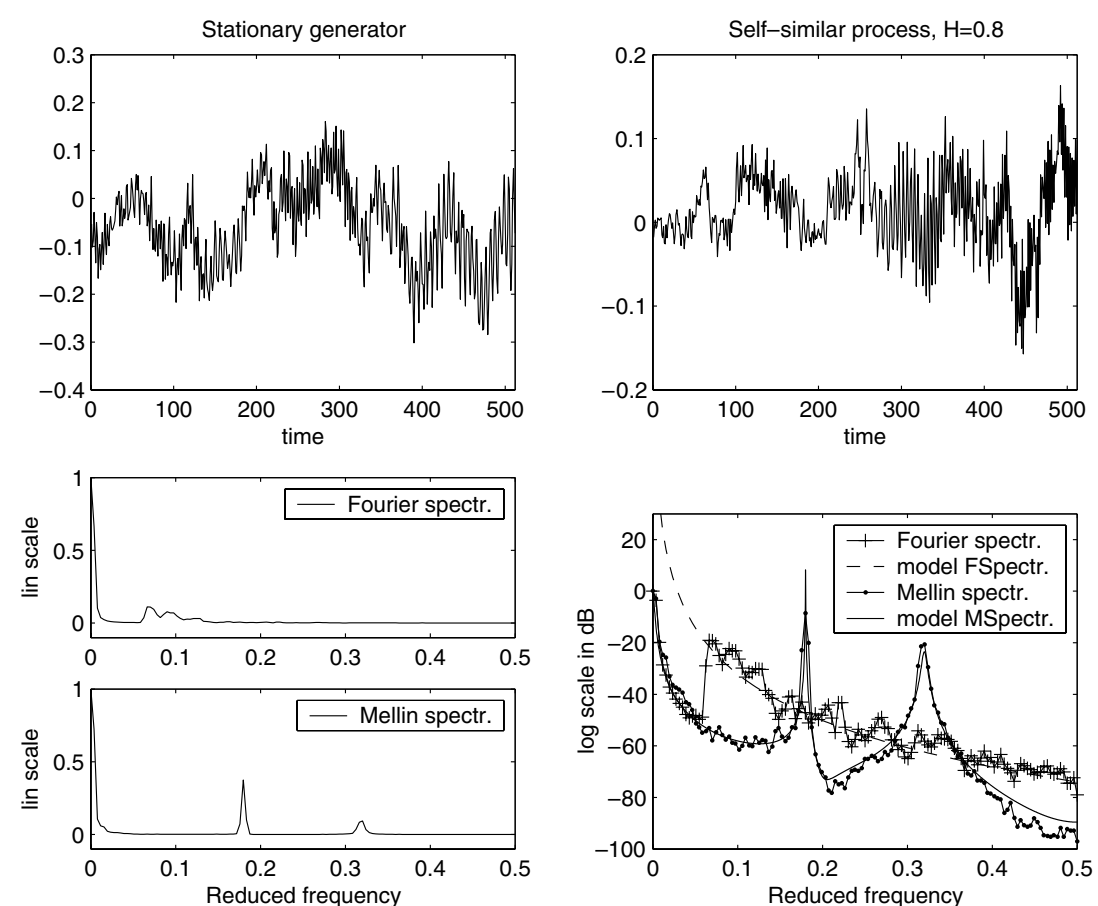

Figure 3. Modelling of a self-similar process with $H=0.8$. Top left: the stationary generator $Y(t)$ is computed as an ARMA model of order $(5,2)$, synthetized from the filtering of a white noise by the transfer function of the ARMA. Top right: the $H$-ss process deduced from the preceding process, implementing numerically $X(t)=\left(\mathcal{L}_{H} Y\right)(t)$. Bottom (left and right): estimated spectrum of $X$ and of its inverse Lamperti transform $Y=\mathcal{L}_{H}{ }^{-1} X$ (done also numerically); left is in linear scale, right in $\log$ scale. The Fourier spectrum of $\mathcal{L}_{H}{ }^{-1} X$ is an estimation of the Mellin spectrum of $X$, according to equation (6). Here, the spectrum of $X$ (which is not properly defined because neither $X$ nor its increments are stationary) appears complex because it has a rather large band and many small resonances and gives only an indication of $H$ by being close to the model $f^{-2 H-1}$ (as shown in the graph). On the other hand, the estimated Mellin spectrum (that is the usual spectrum of $Y$ ) may be modelled well with more details, here an AR(5) whose accurate parameters may be found by an identification on the system (see the model curve on the right).

$T$ is the length of the signal analysed. It has usually small effects. Thus, the relative robustness of the Mellin transform regarding $H$ allows it to be used even if the proper $H$ is not known with great accuracy.

Because using the Lamperti time warping from $t$ to $\log t$ for discrete-time systems is equivalent to using geometric sampling $\left\{q^{n}, n \in \mathbb{Z}\right\}$ for the $H$-ss process (and thus the usual arithmetic process for the stationary generator: $\{n \log q, n \in \mathbb{Z}\}$ ), designing procedures of estimation with geometrical sampling for self-similar processes is equivalent to designing procedures with arithmetic sampling for the stationary generator. This last factor is helped by all the amount of work done on digital signal processing. Consequences of this resampling were examined in specific situations in several works: direct estimation of correlation function $C_{X}$ [32], estimation of the self-similarity index $H$ [54], estimation of spectrum from random geometric sampling [30]. In all cases, the estimation was shown or proved to work. Finally, we have shown that a joint Fourier-Mellin analysis is also possible by means of time-frequency analysis. This method combines both kinds of information: stationarity or non-stationarity in time and stationarity in scale [25]. 


\section{Generalized Lamperti transformations for broken scale invariances}

The equivalence (4) opens a window on generalizations of the Lamperti transformation for broken scale invariance. Indeed, this usual Lamperti correspondence offers perspectives to study any property built on dilations as an image of some property built on shifts: homogeneity, time invariance or even some nonstationarity.

There are two possibilities to extend the relation (4). First, one can suppose some weakened symmetry for the generator besides exact stationarity. For instance, discrete translation invariance (the kind that occurs in a crystal lattice) of the generator leads by $\mathcal{L}_{H}$ to discrete periodicity in scale, and in the next section we will elaborate on this symmetry, known as discrete scale invariance (DSI). This solution draws upon classical results on some classes of nonstationary signals: the properties of cyclostationary signals are mapped to DSI; local stationarity is mapped to local self-similarity (see below).

Another possibility is to make full use of the extended notion of stationarity proposed by Hannan [33]. If stationarity is understood as invariance under any group of transformation $\left\{\mathcal{T}_{g}, g \in G\right\}$ where $G$ is a group (in the case of self-similarity, $\mathcal{T}_{g}$ is a dilation $\mathcal{D}_{H, \lambda}$ and $G$ the multiplicative group $\left(\mathbb{R}_{*}^{+}, \times\right)$), one can ask whether there exists an invertible mapping $\mathcal{L}$ such that $\mathcal{L}^{-1} \mathcal{T}_{g} \mathcal{L}=\mathcal{S}_{\phi(g)}$. A first general answer is that the group $G$ and the group $(\mathbb{R},+)$ should have the same underlying structure, because this relation implies they are isomorphic. We examine the consequences of this remark to study scale invariance with finite-size effects in section 4.

Finally, let us note that reducing any nonstationary process to its stationary generator is addressed from a mathematical point of view (with a strong hypothesis of continuity on the correlation) in geostatistics [49] and statistics [46]. We point out here that the method has practical consequences for the modelling and analysis of scale invariance. Hereafter we deal only with one-dimensional signals, but the study of stationary generators of scale-invariant two-dimensional fields is possible from the same point of view [9].

\subsection{Nonstationary time-scale representations}

General nonstationary methods built on Fourier representation (related to time-shifts) lead to corresponding methods for Mellin representation. As an example, the time-dependent Wigner-Ville spectrum $W_{Y}(t, f)$ [24], where $t$ is the local time and $f$ the local frequency, given by the Fourier transform of the (nonstationary) covariance of the process,

$$
W_{Y}(t, f) \hat{=} \int_{-\infty}^{+\infty} R_{Y}(t+\tau / 2, t-\tau / 2) \mathrm{e}^{-\mathrm{i} 2 \pi f \tau} \mathrm{d} \tau,
$$

is mapped by $\mathcal{L}_{H}$ to a time-Mellin scale representation:

$$
\begin{aligned}
W_{X}^{H \mathrm{ss}}(t, f) & =\int_{0}^{+\infty} R_{X}(t \sqrt{\tau}, t / \sqrt{\tau}) \tau^{-\mathrm{i} 2 \pi f-1} \mathrm{~d} \tau \\
& =t^{2 H} W_{\mathcal{L}_{H}{ }^{-1} X}(\log t, f) .
\end{aligned}
$$

The proof lies in equation (7) that shows that one has to change the Fourier transform for a Mellin one, and consequently that addition of time is replaced by a multiplication in scale. This recovers the scale-invariant Wigner spectrum $W_{X}^{H \text { ss }}(t, f)$ [23], and $f$ has then the meaning of a Mellin scale, which is well adapted to describe invariant properties under dilations as argued before. 
An interest of $W_{Y}(t, f)$ and $W_{X}^{H \text { ss }}(t, f)$ lies in the fact that for a stationary $Y$ process, one recovers via the first one the stationary power spectrum $\Gamma_{Y}(f)$,

$$
W_{Y}(t, f)=\int_{-\infty}^{+\infty} c_{Y}(\tau) \mathrm{e}^{-\mathrm{i} 2 \pi f \tau} \mathrm{d} \tau=\Gamma_{Y}(f),
$$

and for self-similar $X$ process, the corresponding property is that the scale-invariant Wigner spectrum factorizes simply as $W_{X}^{H \text { ss }}(t, f)=t^{2 H} \Gamma_{\mathcal{L}_{H}{ }^{-1} X}(f)$. Note that the scale-invariant Wigner spectrum also has the property of invariance under dilations by a ratio $\lambda$ for any process $X$ (even if it is nonstationary and not $H$-ss):

$$
W_{\mathcal{D}_{H, \lambda} X}^{H \mathrm{ss}}(t, f)=\lambda^{-2 H} W_{X}^{H \mathrm{ss}}(\lambda t, f),
$$

which could be used as a defining property of $W_{X}^{H \text { ss }}(t, f)$.

This representation $W^{H s s}$ gives the evolution in time of a process with respect to scale invariance; thus, it disregards the part of nonstationarity that is linked only to the $t^{H}$ renormalization term of scale invariance. If a signal is scale invariant, one obtains a time-invariant $W^{H s s}$; if not, the time evolution informs about the modifications of the scale composition of the process. Examples of such evolution are given in the following sections.

\subsection{Local self-similarity}

General nonstationary models admit corresponding non-scale-invariant models through $\mathcal{L}_{H}$. A first example is the model for the correlation of locally stationary processes [50] that reads as $R_{Y}(t, s)=m_{Y}\left(\frac{t+s}{2}\right) c_{Y}(t-s)$ with $m_{Y}(t) \geqslant 0$ and $c_{Y}(u)$ a non-negative definite function. The second function is an ordinary stationary covariance whereas $R_{Y}$ fluctuates with the mean local time $(t+s) / 2$ by means of the first function. By Lamperti mapping, a class of non-scale-invariant processes is introduced that has the general covariance

$$
R_{X}(t, s)=m_{X}(\log \sqrt{t s})(t s)^{H} C_{X}(t / s) .
$$

The mapping is obtained from $C_{X}\left(\mathrm{e}^{u}\right)=c_{Y}(u)$ and $m_{X}(t)=m_{Y}(t)$. A comparison with (5) reveals that it is a generalized form allowing some evolution with the local mean time $\sqrt{t s}$ and the corresponding scale-invariant Wigner spectrum expresses as $W_{X}^{H \text { ss }}(t, f)=$ $m_{X}(t) t^{2 H}(\mathbf{M} C)(\mathrm{i} 2 \pi f)$, illustrating both the mean evolution imposed by the function $m_{X}(t)$ and the scale behaviour given by the second term, a local scale spectrum.

We will not detail other aspects, except for discrete scale invariance hereafter, and the reader will find elsewhere details on higher order distributions that can be introduced on this grounding [3], on multiplicative harmonizability for non- $H$-ss processes [10], or on the analysis of locally asymptotically self-similar processes by the use of $\mathcal{L}_{H}[26,11]$.

\section{Discrete scale invariance}

An application of the Lamperti correspondence has received more attention, namely the study of discrete scale invariance, i.e., scale invariance for some preferred scale factors only. Some fractals such as the triadic Cantor set, or some simple signals such as the Mellin chirps of the form $t^{H} \exp \left(\mathrm{i} f_{0} \log t\right)$, are naive examples of this symmetry which is the invariance under dilations of scale factors $\left\{\lambda_{0}^{n}, n \in \mathbb{N}\right\}\left(\lambda_{0}=3\right.$ for a triadic Cantor set and $\lambda_{0}=\exp \left(2 \pi / f_{0}\right)$ for Mellin chirps). This was advocated as a central concept in the study of many critical systems $[47,51,52]$. More attention has been given to the deterministic framework and we rely here on the stochastic extension of the property [10]. 
A process $\{X(t), t>0\}$ is said to possess discrete scale invariance (DSI) of index $H$ and scaling factor $\lambda_{0}>0$ if $\left.\left\{\mathcal{D}_{H, \lambda_{0}} X\right)(t), t>0\right\} \stackrel{d}{=}\{X(t), t>0\}$. Mapping this property back to $Y(t)=\left(\mathcal{L}_{H}{ }^{-1} X\right)(t)$, it is straightforward to establish that, provided that $T_{0}=\log \lambda_{0}$, we have $\left.\left\{\mathcal{S}_{T_{0}} Y\right)(t), t \in \mathbb{R}\right\} \stackrel{d}{=}\{Y(t), t \in \mathbb{R}\}$. This property is a statistical periodicity of $Y$ of period $T_{0}$ (and hence for any time-shift $n T_{0}, n \in \mathbb{N}$ ) and it defines cyclostationary (or periodically correlated) processes $[29,31]$. The Lamperti correspondence is such that a process has $\left(H, \lambda_{0}\right)$-DSI if and only if its inverse Lamperti transform is cyclostationary of period $T_{0}=\log \lambda_{0}$. This is an extension to stochastic DSI of the Lamperti result on $H$-ss.

\subsection{Characterization of DSI}

The theory of cyclostationary processes is well established [28]. A known characterization uses the periodicity of the covariance, $R_{Y}(t+T, s+T)=R_{Y}(t, s)$, to write it as a Fourier series

$$
R_{Y}(t, t+\tau)=\sum_{n=-\infty}^{+\infty} c_{n}(\tau) \mathrm{e}^{\mathrm{i} 2 \pi n t / T_{0}}
$$

A corresponding first characterisation of DSI is thus obtained on the covariance which has to read for a process $X(t)$ with $\left(H, \lambda_{0}\right)$-DSI

$$
R_{X}(t, k t)=(k t)^{H} \sum_{n=-\infty}^{+\infty} c_{n}(\log k) t^{H+\mathrm{i} 2 \pi n / \log \lambda_{0}} .
$$

Furthermore, using time-Mellin scale analysis, one obtains a simple expansion of the covariance on Mellin chirps.

$$
W_{X}^{H \mathrm{ss}}(t, f)=\sum_{n}\left(\mathbf{F} c_{n}\right)\left(f-\frac{n}{\log \lambda_{0}}\right) t^{2 H+\mathrm{i} 2 \pi n / \log \lambda_{0}} .
$$

This equation offers direct possibilities of studies of processes with DSI in a time-frequency space. Any DSI signal may be thus decomposed on a Mellin chirp expansion.

\subsection{Discrete-time sequences with DSI}

An important feature of stochastic DSI is that one can analyse discrete-time sequences that might have this property by using standard cyclostationary tools on the stationarized process. Estimation of the cyclostationary period of this process, e.g., by means of the marginal of the cyclic periodogram, will give an estimate of the preferred scale ratio $\lambda_{0}$. From equation (21), a weighted time average on the Mellin functions reads

$$
\int_{0}^{\infty} W_{X}^{H \mathrm{ss}}(t, f) t^{-2 H-\mathrm{i} 2 \pi \beta_{c}-1} \mathrm{~d} t=\sum_{n}\left(\mathbf{F} c_{n}\right)\left(f-\frac{n}{\log \lambda_{0}}\right) \delta\left(\beta_{c}-n / \log \lambda_{0}\right) .
$$

Taking then the sum over all the scales $f$ leads to the marginal cyclic spectrum that should show peaks on specific Mellin scales related to the preferred scale ratio:

$S\left(\beta_{c}\right)=\int_{-\infty}^{+\infty} \mathrm{d} f \int_{0}^{\infty} W_{X}^{H \mathrm{ss}}(t, f) t^{-2 H-\mathrm{i} 2 \pi \beta_{c}-1} \mathrm{~d} t=\sum_{n} E_{n} \delta\left(\beta_{c}-n / \log \lambda_{0}\right)$.

Here, $E_{n}=\int_{-\infty}^{+\infty} \boldsymbol{\Gamma}_{n}(f) \mathrm{d} f=\int_{-\infty}^{+\infty}\left(\mathbf{F} c_{n}\right)(f) \mathrm{d} f$ is, thanks to the Wiener-Khintchin relation, the total energy of a process having $c_{n}(\tau)$ as correlation function. Thus, there are peaks in this cyclic spectrum, localized on Mellin scales $\beta_{c}=n / \log \lambda_{0}$. 

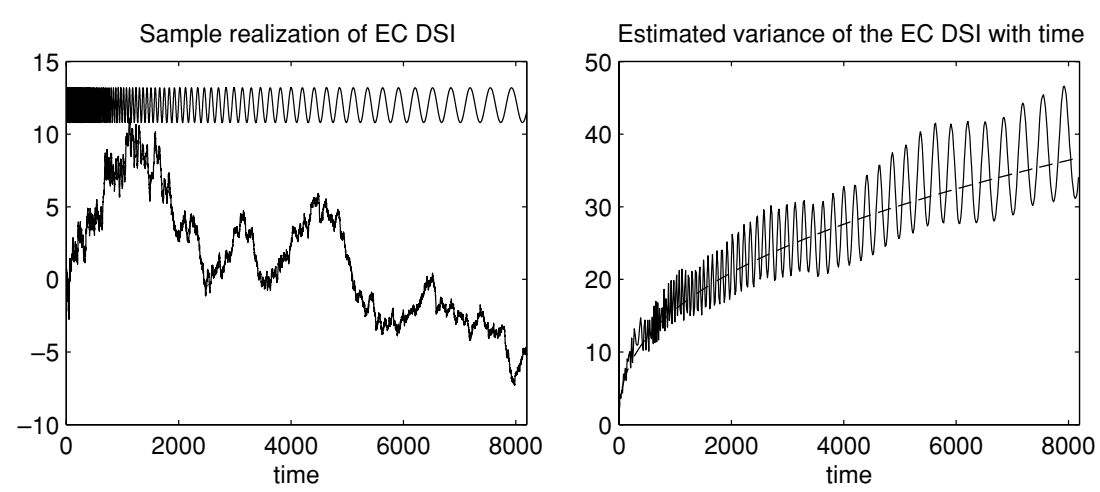

Figure 4. Example of parametric system with discrete scale invariance. The model is based on a time-varying scale invariant filter (or Euler-Cauchy system), with a time representation as $t X^{\prime}(t)+a(t) X(t)=\epsilon(t) . \epsilon(t)$ is white noise with time-varying variance $\left(t^{H} \sigma(t)\right)^{2}$ and we impose that $a(t)$ and $\sigma(t)$ have deterministic DSI with exponent 0 , i.e., $a\left(\lambda_{0} t\right)=a(t)$. This model, known as a DSI Euler-Cauchy system of order 1, is studied in details in [11], along with its proper discretisation in time. Left: sample path of the process with $H=0.2$ and $\lambda_{0}=1.05$; a figuration of the oscillations of the coefficients is shown above it. Right: estimated variance of the process, done empirically with 1024 realizations. The log-periodic oscillations of the variance are a signature of DSI for this model.

In addition, there exist various models, parametric or not, of random sequences having DSI [11] that may serve as benchmarks. As an example, we plot in figure 4 one realization of a stochastic process with DSI, with its estimated variance on the right. If one wants to resort to cyclostationary methods for the DSI problem for instance, a statistical comparison of the estimates found for some real data and the results obtained on sequences with or without DSI will escape the difficulties and the possibilities of artefacts regarding DSI studied in [34].

\subsection{DSI and stationary increments}

The property of DSI is compatible with the property of stationary increments. This is known since studies on the Weierstrass-Mandelbrot random function [7, 25]:

$$
W(t)=\sum_{n=-\infty}^{+\infty} \lambda_{0}^{-n H}\left(1-\mathrm{e}^{\mathrm{i} \lambda_{0}^{n} t}\right) \mathrm{e}^{\mathrm{i} \phi_{n}},
$$

where the $\phi_{n}$ are i.i.d. random variables, uniform in $[0,2 \pi$ [. An immediate property is the invariance of $W(t)$ under dilations $\mathcal{D}_{H, \lambda}$, but only of $\lambda=\lambda_{0}^{m}$ with $m \in \mathbb{Z}$, that is DSI. Analysed as such, the Weierstrass-Mandelbrot random functions admit a decomposition on a Mellin basis. In figure 5, we illustrate this property by showing a sample realization of this random process and the corresponding cyclic Mellin spectrum that was defined in (23). A less known property concerns the increment process and reads

$$
\mathbb{E}|W(t+\tau)-W(t)|^{2}=\sum_{n=-\infty}^{+\infty} \lambda_{0}^{-2 n H} 2\left(1-\cos \lambda_{0}^{n} \tau\right)
$$

This expression is given by a straightforward calculus on the possible random phases. A striking feature is that the structure function of order 2 of $W(t)$ does not depend on $t$ but only on $\tau$; that is known as (second-order) stationary increments. This property is interesting because it allows a proper definition to the Fourier spectrum of this otherwise non-stationary process to be given. 

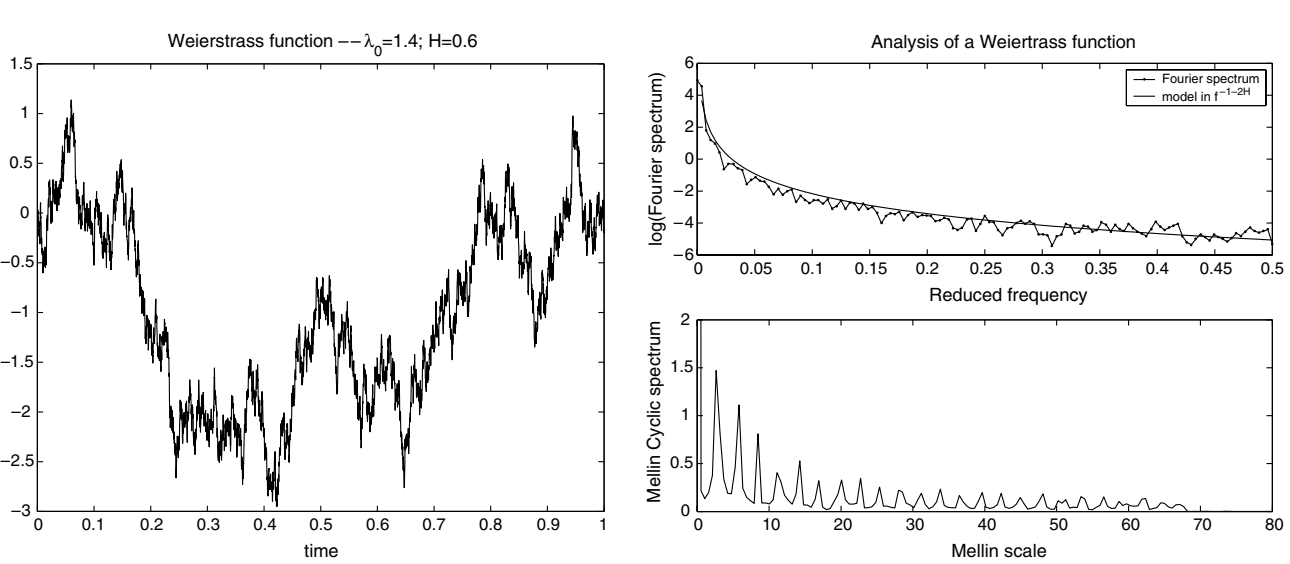

Figure 5. Example of a random Weierstrass-Mandelbrot function, as defined in equation (24) on the left, with $H=0.4$ and $\lambda_{0}=1.4$. The simulation is made with a limited number of modes, the ones of higher frequency being $n=50$. Elements of analysis are shown on the right by means of $\left(\mathcal{L}_{H}{ }^{-1} W\right)(t)$. Upper part: an estimate of the Fourier spectrum of $\left(\mathcal{L}_{H}{ }^{-1} W\right)(t)$, superimposed with the long-range dependence model for the spectrum in $f^{-1-2 H}$ - this spectrum shows no striking feature of preferred scale ratio; lower part: an estimate of the cyclic spectrum $S\left(\beta_{c}\right)$ as defined in (23) is represented. The estimation was first made by doing numerically an inverse Lamperti transform, and then a double Fourier transform is made to estimate the quantity appearing in (22). Finally, a sum is made on the frequency $f$. An average was also made by cutting the time series into several blocks and averaging the result over those blocks. The peaks are separated in Mellin scale by $1 / \log \lambda \simeq 2.97$. For an exactly self-similar process, one would find only a peak around $\beta_{c}=0$, so they are here relevant to measure the DSI.

This process can also be used as a starting model for DSI processes with stationary increments, changing $\left(1-\mathrm{e}^{\mathrm{i} \lambda_{0}^{n} t}\right)$ in its expression for a more general form $\left(g(0)-g\left(\lambda_{0}^{n} t\right)\right)$, with any periodic function continuously differentiable at $t=0$. DSI and some kind of stationarity might then coexist in physical models needing both. The reader is referred to [25] for a more detailed lecture on the properties on the Weiertrass-Mandelbrot function and the possibilities of uncovering its properties by time-frequency methods.

\section{Warped Lamperti transformation for broken self-similarity}

We have said that the Lamperti transform can be adapted to other forms of symmetry than proper scale invariance, by modifying $\mathcal{L}_{H}$ so that an equivalence similar to (4) remains valid. An interesting approach to tackle broken scale invariant signals is to postulate that the usual composition of scales $(\lambda \odot t=\lambda \times t)$ is no longer valid, and that another way of composing scales underlies the physics: this postulates an unusual law and action on scales. A symmetry of this kind was first adopted by Nottale in his theory of scale relativity [43], and further developed by Dubrulle and Graner in another context [17, 18]. The symmetry in scale is broken because of the existence of bounds in scale and amplitude of the studied processes.

We present here a general setting that allows us to put in correspondence stationary stochastic processes with generalized scale-invariant processes with finite size effects or bounding. The setting is presented before constructing the generalized Lamperti transform associated with this law, and we give details on finite-size scale-invariant processes in section 4.3. This generalized stationarizing transform gives new insights into this symmetry because it opens the subject to results coming from stationary modelling. 


\subsection{Generalized scale laws and the associated dilation operator}

The stochastic processes $X(t)$ we consider describe the evolution in scale of some physical quantities. Therefore, the transformation of scales $\mathrm{e}_{1} \odot \mathrm{e}_{2}=\mathrm{e}_{3}$ and the transformation of the process must belong to a group of transformations (or at least to a semi-group, such as happens in multiplicative cascades [14]).

We restrict the discussion here to the case for which scales belong to a group. Behind the hypothesis of an invertible law for the scales, the idea is that changing scale is an operation meaningful both as zooming out and zooming in so that each zooming ratio has to possess an inverse. The simplest choice is to take a group isomorphic to $\left(\mathbb{R}^{+}, \times\right)$. Let $\mathbb{A}$ be the set of scales that we consider and let $\odot$ be the law of composition of scales. Since we postulate that $(\mathbb{A}, \odot)$ is isomorphic to $\left(\mathbb{R}_{*}^{+}, \times\right)$, there exists a morphism $S_{\odot}: \mathbb{A} \longrightarrow \mathbb{R}_{*}^{+}$such that $S_{\odot}\left(\mathrm{e}_{1} \odot \mathrm{e}_{2}\right)=S_{\odot}\left(\mathrm{e}_{1}\right) \times S_{\odot}\left(\mathrm{e}_{2}\right)$. Any diffeormorphism from $\mathbb{A}$ onto $\mathbb{R}^{+}$is a good candidate and for any $\alpha \in \mathbb{R},\left(S_{\odot}(\cdot)\right)^{\alpha}$ is also a convenient morphism.

A similar hypothesis is made on the amplitude of the process. For instance, instead of a process valued in $\mathbb{R}$, we may assume that it takes values in an interval $\mathbb{X}=] X_{-}, X_{+}[$included in $\mathbb{R}_{*}^{+}$. A discussion concerning this restriction is provided below. We will show later that there exists a law $\otimes$ of composition on this interval that makes $(\mathbb{X}, \otimes)$ a group isomorphic to $\left(\mathbb{R}_{*}^{+}, \times\right)$. Let $S_{\otimes}: \mathbb{X} \longrightarrow \mathbb{R}_{*}^{+}$be the associated morphism. Note here that the morphism $S_{\otimes}$ depends explicitly on the bounds of interval $\mathbb{X}$ : in this formalism, we study finite size effects.

We can now define a generalized dilation operator following the construction of the usual dilation operator: time is dilated using the composition of scales $(x)$ and the process is renormalized properly using the composition law for amplitudes $(x)$. Let $\lambda$ be a dilation factor in $\mathbb{A}$. We then define the generalized dilation operator $\mathcal{D}_{H, \lambda}^{g}$, acting on stochastic processes indexed by $\mathbb{A}$ with values in $\mathbb{X}$, as

$$
\left(\mathcal{D}_{H, \lambda}^{g} X\right)(t)=g(\lambda) \otimes X(\lambda \odot t),
$$

where $g(\lambda)$ is the renormalization function. This function is not arbitrary and indeed depends on the morphisms $S_{\odot}$ and $S_{\otimes}$. To prove this, we note that going from time $t$ to time $\lambda_{1} \odot \lambda_{2} \odot t$ can be done at least in two ways: directly by applying $\mathcal{D}_{H, \lambda_{1} \odot \lambda_{2}}^{g}$ or indirectly by applying successively $\mathcal{D}_{H, \lambda_{1}}^{g}$ and $\mathcal{D}_{H, \lambda_{2}}^{g}$. Mathematically, this means that the generalized dilation operator is a representation of the group $(\mathbb{A}, \odot)$. It also implies that the function $g$ satisfies $g\left(\lambda_{1} \odot \lambda_{2}\right)=g\left(\lambda_{1}\right) \otimes g\left(\lambda_{2}\right)$, meaning that $S_{\otimes} \circ g=S_{\odot}{ }^{\alpha}$ is an acceptable morphism for $\odot$. For reasons that will be clear later, we choose the exponent $\alpha=-H$ so that $g(\lambda)=S_{\otimes}^{-1}\left(S_{\odot}(\lambda)^{-H}\right)$.

Given the notions introduced above, we will say that a stochastic process satisfies a generalized scale-invariance property if

$$
X(t) \stackrel{d}{=}\left(\mathcal{D}_{H, \lambda}^{g} X\right)(t)=g(\lambda) \otimes X(\lambda \odot t) .
$$

This equation is directly a generalization of (1). Note that imposing the equality for a deterministic function $x(t)$ allows us to obtain the form of the scale-invariant function (analogy with power laws for the usual dilation operator). Indeed, a scale-invariant function satisfies $x(t)=S_{\otimes}^{-1}\left(S_{\odot}(\lambda)^{-H}\right) \otimes x(\lambda \odot t), \forall \lambda \in \mathbb{A}$. Let $e$ be the identity element for $\odot$. Then setting $\lambda$ so that $\lambda \odot t=e$ (the group structure implies the existence of the inverse $\lambda=e \odot^{-1} t$ ) allows us to write

$$
\begin{aligned}
x(t) & =x(e) \otimes S_{\otimes}^{-1}\left(S_{\odot}\left(e \odot^{-1} t\right)^{-H}\right) \\
& =x(e) \otimes S_{\otimes}^{-1}\left(S_{\odot}(t)^{+H}\right)=S_{\otimes}^{-1}\left[S_{\otimes}(x(e)) S_{\odot}(t)^{H}\right] .
\end{aligned}
$$


This expression has two free parameters once the morphisms are fixed: the exponent $H$ and the multiplicative constant $S_{\otimes}(x(e))$ that defines the initial value. In the usual case, $\odot=\times$ and the morphisms are the identity. We recover the usual power law as the deterministic scale-invariant function and $g(\lambda)=\lambda^{-H}$. This recovers expression (1) for the dilation and this explains the choice $\alpha=-H$ previously adopted.

\subsection{Generalized Lamperti transformation}

We have shown in the previous sections the usefulness of the Lamperti transformation to study scale invariant processes (and their broken versions). The fundamental fact behind this is that dilation and shift operators are equivalent through $\mathcal{L}_{H}$, according to (4). The same idea can be applied for generalized dilation operators. We hence seek an operator $\mathcal{L}_{H}^{g}$, acting on stationary signals $Y$ indexed by $\mathbb{R}$ such that $\left(\mathcal{L}_{H}^{g} Y\right)(t)$ satisfies the generalized scale-invariance property. This operator must be invertible, and generalized dilation and shift should be equivalent through its application. The expressions for the generalized Lamperti transformation and its inverse are easily shown to be

$$
\begin{aligned}
\left(\mathcal{L}_{H}^{g} Y\right)(t) & =S_{\otimes}^{-1}\left(Y\left(\log S_{\odot}(t)\right)\right) \otimes S_{\otimes}^{-1}\left(S_{\odot}(t)^{H}\right) \\
& =S_{\otimes}^{-1}\left(S_{\odot}(t)^{H} Y\left(\log S_{\odot}(t)\right)\right) \\
\left(\mathcal{L}_{H}^{g-1} X\right)(t) & =\mathrm{e}^{-H t} S_{\otimes}^{-1}\left(X\left(S_{\odot}^{-1}\left(\mathrm{e}^{t}\right)\right)\right)
\end{aligned}
$$

making $\mathcal{D}_{H, \lambda}^{g}$ and $\mathcal{S}_{\log S_{\odot}(\lambda)}$ equivalent operators, since

$$
\mathcal{L}_{H}^{g}{ }^{-1} \mathcal{D}_{H, \lambda}^{g} \mathcal{L}_{H}^{g}=\mathcal{S}_{\log S_{\odot}(\lambda)} .
$$

The structure of the generalized Lamperti transformation is interesting, since it can also be written as a function of the usual Lamperti transformation. Indeed, we have

$$
\left(\mathcal{L}_{H}^{g} Y\right)(t)=S_{\otimes}^{-1}\left(\left(\mathcal{L}_{H} Y\right)\left(S_{\odot}(t)\right)\right) .
$$

Therefore, a signal that has a generalized scale-invariance property can be constructed from its stationary generator or can be obtained by time and amplitude warping of the associated $H$-ss process.

Note that the morphisms are indeed deterministic functions. We could imagine replacing in the definition above deterministic morphisms by random morphisms (Devil's staircase associated with a random measure, for example). In this way, we recover approaches taken by processes with multifractal times [40] (see also [14] for more recent applications) in some definitions of multifractal processes. The link between the ideas developed here and multifractals remains however to be explored.

\subsection{Finite-size scale-invariant fractional Brownian motion}

As an illustration of the ideas presented above, we consider the approach initiated by Notalle in his theory of scale relativity [43], and further developed by Dubrulle and Graner [18, 17]. In these works, it is argued that the law for composition of scales may be more complicated than the usual product. Scale has the behaviour of a velocity when considered logarithmically, as $a=\log \lambda$. A natural generalization is to consider that the logarithm of the scale does not follow the Galilean transformation of velocities but the Lorentz law. In this setting, scale is limited to a finite size range, and Dubrulle has developed the formalism of finite size scale invariance using this. 
However, this scale invariance was applied directly to deterministic functions, or to the moments of random variables. As the invariance is only studied as a possible model for those moments, the method does not give tools of synthesis or of analysis beyond testing the goodness-of-fit of the finite-size scaling laws for some data. Our approach is slightly different in that we impose scale invariance on the random variables themselves. This results in probabilistic scale invariance where the statistics will follow deterministic laws of finitesize scaling studied by Dubrulle; but as we work jointly on the scale evolution of the process and its probability law by means of a Lamperti correspondence with a stationary generator, we obtain general methods to synthesize and analyse those processes. Our framework is more constrained, but gives better insights into the studied stochastic processes because of the correspondence with stationary processes. Thus, there exists the possibility of adapting methods of stationary signal processing to finite-size scale invariance.

If scale is constrained to live in the finite size interval, let $\mathbb{A}=] a_{-}, a_{+}\left[\subseteq \mathbb{R}_{*}^{+}\right.$. Then, the Lorentz composition law for two scales reads

$$
a_{1} \odot a_{2}=\exp \frac{\log a_{1}+\log a_{2}-\log a_{1} \log a_{2}\left(\frac{1}{\log a_{-}}+\frac{1}{\log a_{+}}\right)}{1-\frac{\log a_{1} \log a_{2}}{\log a_{-} \log a_{+}}} .
$$

The associated morphism can be shown to be

$$
S_{\odot}(a)=\left\{\begin{array}{l}
\exp \left\{\frac{\log a_{-} \log a_{+}}{\log a_{-}-\log a_{+}} \log \left(\frac{1-\log a / \log a_{-}}{1-\log a / \log a_{+}}\right)\right\} \\
\exp \left\{\log \frac{1}{a_{ \pm}} \log \left(1-\frac{\log a}{\log a_{ \pm}}\right)\right\}, \quad \text { if } \quad \log a_{\mp} \rightarrow+\infty . \\
a, \quad \text { if furthermore } \log a_{ \pm} \longrightarrow-\infty
\end{array}\right.
$$

The same form of composition laws is adopted for the amplitudes of the signals, leading to the same form of morphism, replacing variables $a$ by $X$ in $\mathbb{X}=] X_{-}, X_{+}[$. There is however a difficulty concerning the application of this formalism to the amplitude of the signals: these laws are based on the equivalence between the multiplicative and the additive groups via a logarithm. Therefore, they are restricted, as mentioned above, to the case of positive variables. This restriction is not a real problem for time, since we are satisfied to work with signals indexed by $\mathbb{R}_{*}^{+}$only. It is more problematic when considering the amplitude of the signals that can be either positive or negative. To manipulate signed signals, the composition law has to separate the positive part from the negative part of the amplitude. Hence, the elements of the groups are represented as two-parameter elements $X=(|X|, \operatorname{Sign}(X))$. In this case, the morphism is defined as

$$
\begin{aligned}
& S_{\otimes}:\left(\mathbb{X}^{*}, \otimes\right) \longrightarrow\left(\mathbb{R}^{*}, \times\right) \\
& X \longrightarrow S_{\otimes}(X)=\theta S_{\theta}(|X|) \quad \text { where } \quad \theta=\operatorname{Sign}(X)
\end{aligned}
$$

and where $S_{+1}$ (resp. $S_{-1}$ ) is a function between ]0, $X_{+}$[ (resp. ]0, $X_{-}$[) onto $\mathbb{R}^{+*}$. In the case of the special relativity-like laws, the details of the laws can be found in [2], and the functions $S_{\theta}$ are given by

$$
\begin{cases}S_{+1}(X)=\exp \left(-\log X_{+} \log \left(1-\frac{\log |X|}{\log X_{+}}\right)\right) & \text {if } \quad X \geqslant 0 \\ S_{-1}(X)=\exp \left(-\log X_{+} \log \left(\frac{\log X_{-}-\log |X|}{\log X_{+}}\right)\right) & \text {if } \quad X<0 .\end{cases}
$$

The inverse of the morphism is given by

$$
\begin{aligned}
& S_{\otimes}^{-1}:\left(\mathbb{R}^{*}, \times\right) \longrightarrow\left(\mathbb{X}^{*}, \otimes\right) \\
& x \longrightarrow S_{\otimes}^{-1}(x)=\theta S_{\theta}^{-1}(|x|) \quad \text { where } \quad \theta=\operatorname{Sign}(x) .
\end{aligned}
$$


There is no real separation between the positive and the negative part; the morphism remains continuous in 0 and we may set $S_{\otimes}(0)=0$. The separation was made to respect the group structure, and to obey the fact that 0 has a specific role with regards to the multiplication. The only specificity left is the fact that the valid scale ratios are taken positive only (because here the time is positive only), so there is no way for a dilation to change the sign of a function. But as the equality is imposed in law, a given realization of a process is allowed to explore without restriction both the positive and the negative values.

These results allow us to study a generalization of the fractional Brownian motions; the stationary generator of the $\mathrm{fBm}$ (generalized Ornstein-Uhlenbeck process, gOU), whose correlation function is given by (12), can be used to create an $\mathrm{fBm}$ with a generalized scaleinvariance property, with finite-size effects. Using the explicit forms of the morphisms corresponding to the finite-size scale composition law provided above, we define the finitesize scale-invariant fractional Brownian motion as the generalized Lamperti transform of the gOU. We plot in figure 6 several examples depending on the finiteness of the bounds in scale (and thus in time) and/or in amplitude. According to equation (34), the finite-size scaleinvariant process can be obtained directly from the scale-invariant process sharing the same stationary generator. Therefore, the different snapshots in the figure are obtained by applying the warpings to numerically generated $\mathrm{fBms}$ (using the middle point displacement method, even if the snapshots obtained with this method are just approximations of $\mathrm{fBm}$ ). Some of the snapshots have a strange look, and their usefulness to describe real processes is of course under question (and study). To assess the usefulness of some of the models, we could imagine that the fssi-fBm bounded in time and unbounded in amplitude could model a critical phenomenon presenting a rupture. In this view, the upper bound $a_{+}$could represent the critical time.

The complete study of the processes is quite difficult to perform. When there is no warping in amplitude, the study is easy since the only transformation acts on time. Hence, the $\mathrm{fBm}$ with a generalized scale-invariant property (restricted to scale) is a Gaussian process with covariance directly obtained from that of the fBm by properly warping the time lags of the covariance function. From equation (11), the covariance reads

$$
R(t, s)=\frac{\sigma^{2}}{2}\left(\left|S_{\odot}(t)\right|^{2 H}+\left|S_{\odot}(s)\right|^{2 H}+\left|S_{\odot}(t)-S_{\odot}(s)\right|^{2 H}\right)
$$

and fully describes the process. In particular, the variance of the process reads $\sigma^{2}\left|S_{\odot}(t)\right|^{2 H}$, close to the model studied in $[18,17]$ for moments of a finite-size process. When the scale is far from the bounds, the morphism is close to a power law: $\left|S_{\odot}(t)\right|^{2 H} \simeq t^{2 H}$. We obtained thus a form of intermediate asymptotic [5] with a specific departure from the power law given by the morphism law $S_{\odot}$.

When a warping of the amplitude is present, the probabilistic structure can be obtained but is practically restricted to a few point statistics. For example, let $Z$ be a scale-invariant process with stationary generator $Y$. Since $Y$ is a stationary signal, its one point probability density function $P_{Y}$ does not depend on time. Therefore, the one point probability density function of $Z$ reads $P_{Z}(z, t)=P_{Y}\left(z / t^{H}\right) /|t|^{H}$. Therefore, the one point probability density function of $X$ reads

$$
P_{X}(x, t)=\frac{1}{\left|S_{\odot}(t)\right|^{H}} P_{Y}\left(\frac{\theta S_{\theta}(x)}{\left|S_{\odot}(t)\right|^{H}}\right)\left|\frac{\mathrm{d} S_{\theta}(x)}{\mathrm{d} x}\right|,
$$

where again $\theta=\operatorname{Sign}(x)$. This departs from the framework of [17], because we characterize the probability law directly. As an illustration, we plot in figure 7 two of these functions for 

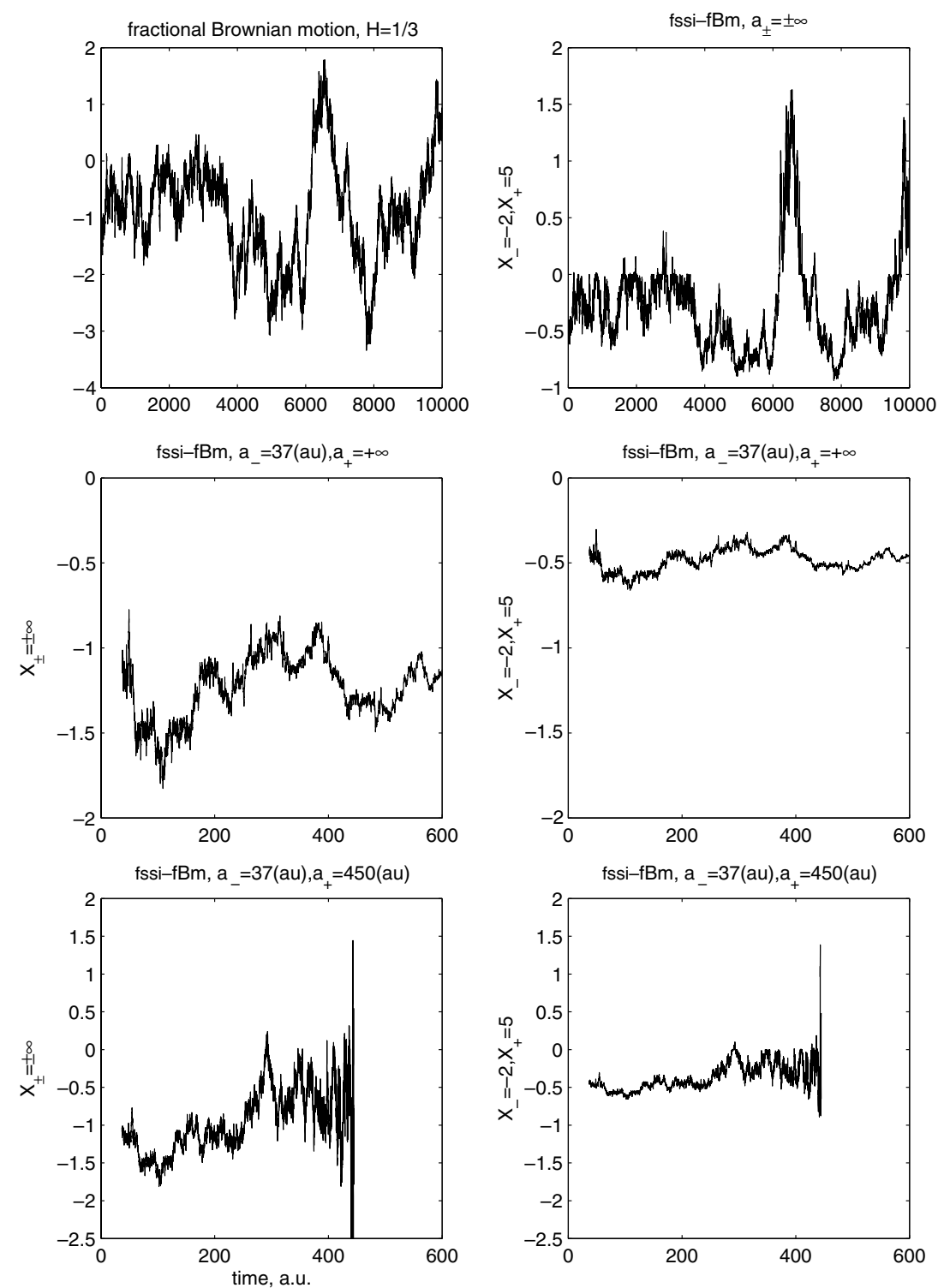

Figure 6. Example of fractional Brownian motion with a finite-size scale-invariance property. Top left: an $\mathrm{fBm}$ with $H=1 / 3$, Hölder exponent for the velocity in fully developed turbulence. Middle left: same fBm after a time warping, time is bounded below. Bottom left: same fBm but warped to a finite-size interval. Top right: same $\mathrm{fBm}$ but warped in amplitude between -2 and 5. Middle right: same fBm after an amplitude warping between -2 and 5 , and bounded below in time. Bottom right: same as middle right but defined on a finite interval of time. The top-left panel corresponds to the usual self-similar processes, whereas the other five depict snapshots of processes that possess some kind of finite-size scale invariance (fssi).

$H=1 / 2$ : in the first case, we choose $-X_{-} \neq X_{+}$, implying that the nonlinear distortion is asymmetric. The nonlinear function is depicted in the top-left figure, whereas the Gaussian and the density of the transformed process are plotted in the top-right figure. The symmetric case is depicted in the bottom row of the figure. 

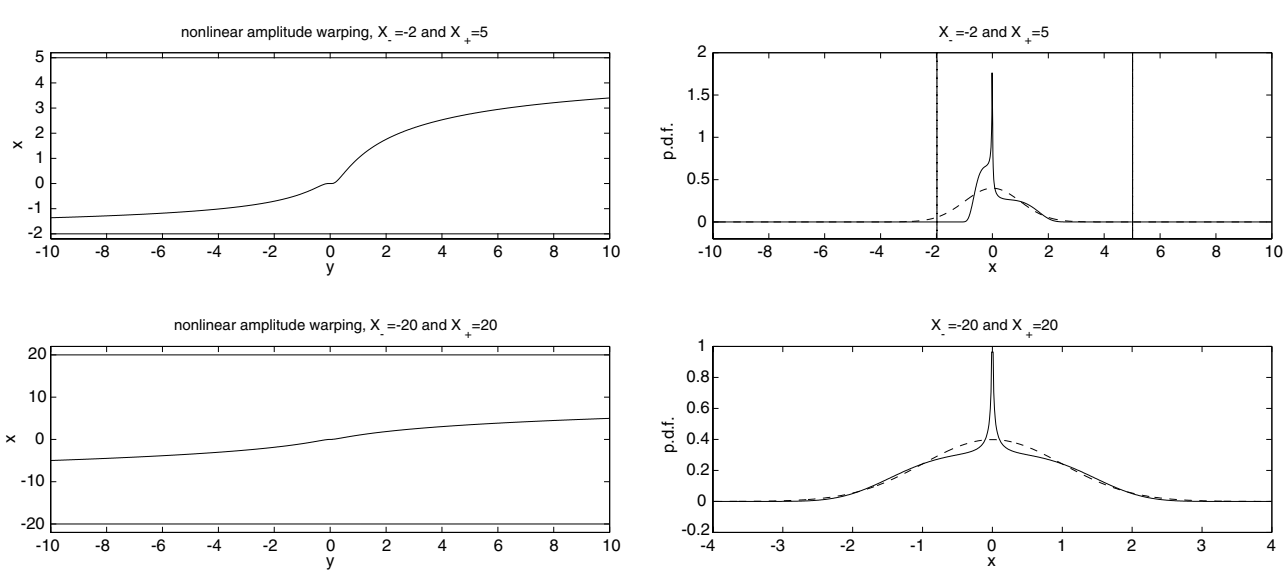

Figure 7. The figures on the left depict the static nonlinearity used to warp the amplitude of the signals, for two cases. The figures on the right give the corresponding probability density functions obtained, when the initial signal is Gaussian.

\section{Conclusion}

We have addressed here some reflections to use the Lamperti correspondence and the stationary generators of self-similar processes, but a furthermore important feature is the existence of enlarged correspondences with broken or weakened self-similarities. An incentive to delve further into the practical use of this framework is that some specific examples, namely discrete scale invariance and finite-size scale-invariance, were put forward in previous works as relevant properties of physical systems: geophysics, fracture and growth problems for the former $([19,52]$ and references herein), turbulence or fundamental physics for the latter [43, 17].

Here, our point of view was stochastic modelling and signal processing. We have paved the way by showing the general framework of stationary correspondence and generators, and given some detailed consequences. From this point of view, numerical models have been obtained and characterized that have the broken scale invariance envisioned here. The future of this work is to study specific physical systems and signals therefrom by means of the methods constructed with the Lamperti transformation, in order to find, or rule out, the appearance of broken scale invariance in problems of physics.

\section{Acknowledgments}

PB would like to acknowledge the support of an INRIA post-doctoral fellowship at the IST, ISR (Lisbon, Portugal) during the course of this work.

\section{References}

[1] Abry P, Flandrin P, Taqqu M and Veitch D 2000 Wavelets for the analysis, estimation, and synthesis of scaling data Self-Similar Network Traffic and Performance Evaluation ed K Park and W Willinger (New York: Wiley) pp 39-88

[2] Amblard P-O, Borgnat P and Flandrin P 2003 Stochastic processes with finite size scale invariance SPIE Conf. on Noise and Fluctuations (Santa Fe, USA, June 2003) vol 5114 pp 161-72

[3] Amblard P-O and Michel O 1999 Vers des distributions affines multilinéaires Coll. GRETSI-99 pp 19-22

[4] Baraniuk R and Jones D 1994 Warped wavelet bases: unitary equivalence and signal processing Proc. IEEE ICASSP-93 (March 1994) vol III pp 320-3 
[5] Barenblatt G 1996 Scaling, Self-similarity, and Intermediate Asymptotics (Cambridge: Cambridge University Press)

[6] Beran J 1994 Statistics for Long-Memory Processes (New York: Chapman \& Hall)

[7] Berry M and Lewis Z 1980 On the Weierstrass-Mandelbrot fractal function Proc. R. Soc. Lond. A 370 459-84

[8] Bertrand J, Bertrand P and Ovarlez J P 1990 Discrete Mellin transform for signal analysis Proc. IEEE ICASSP-90 pp 1603-6

[9] Borgnat P 2004 Symétrie des champs bidimensionnels et générateurs stationnaires Méthodes pour les signaux complexes en traitement d' image ed H Yahia pp 23-30

[10] Borgnat P, Flandrin P and Amblard P-O 2002 Stochastic discrete scale invariance IEEE Signal Process. Lett. 9 $181-4$

[11] Borgnat P 2002 Modèles et outils pour les invariances d'échelle brisées: variations sur la transformation de Lamperti et contributions aux modèles statistiques de vortex en turbulence $P h D$ Thesis École normale supérieure de Lyon

[12] Braccini C and Gambardella G 1986 Form-invariant filtering: theory and applications IEEE Trans. Acoustics Speech Signal Process. 34 1612-27

[13] Burnecki K, Maejima M and Weron A 1997 The Lamperti transformation for self-similar processes Yokohama Math. J. 44 25-42

[14] Chainais P, Riedi R and Abry P 2003 Scale invariant infinitely divisible cascades Int. Symp. on Physics in Signal and Image Processing (Grenoble, France, January 2003)

[15] Daubechies I 1992 Ten Lectures on Wavelets (Philadelphia, PA: SIAM)

[16] Doob J 1967 Stochastic Processes (New York: Wiley)

[17] Dubrulle B 2000 Finite scale size invariance Eur. Phys. J. B 14 757-71

[18] Dubrulle B and Graner F 1996 Possible statistics of scale invariant systems J. Phys. II France 6 797-816

[19] Dubrulle B, Graner F and Sornette D (ed) 1997 Scale Invariance and Beyond (Berlin: EDP Sciences-Springer)

[20] Embrechts P and Maejima M 2002 Selfsimilar processes (Princeton, NJ: Princeton University Press)

[21] Falconer K 1990 Fractal Geometry (New York: Wiley)

[22] Fisher M 1983 Scaling, universality and renormalization group theory Critical Phenomena (Lecture Notes in Physics vol 186) ed F J W Hahne (Berlin: Springer) pp 1-139

[23] Flandrin P 1990 Scale-invariant Wigner spectra and self-similarity Signal Processing V: Theories and Applications ed L Torres et al (Amsterdam: Elsevier) pp 149-52

[24] Flandrin P 1999 Time-Frequency/Time-Scale Analysis (New York: Academic)

[25] Flandrin P and Borgnat P 2003 On the chirp decomposition of Weierstrass-Mandelbrot functions, and their time-frequency interpretation Appl. Comput. Harmon. Anal. 15 134-46

[26] Flandrin P, Borgnat P and Amblard P-O 2003 From stationarity to self-similarity, and back: variations on the Lamperti transformation Processes with Long-Range Correlations: Theory and Applications (Lecture Notes in Physics vol 621) ed G Raganjaran and M Ding (Berlin: Springer) pp 88-117

[27] Frisch U 1995 Turbulence (Cambridge: Cambridge University Press)

[28] Gardner W 1986 The spectral correlation theory of cyclostationary time-series Signal Process. 11 13-36

[29] Gardner W and Franks L 1975 Characterization of cyclostationary random signal processes IEEE Trans. Inforum. Theory 21 4-14

[30] Girardin V and Rachdi M 2003 Spectral density estimation from random sampling for multiplicative stationary processes Comput. Math. Appl. 46 1009-21

[31] Gladyshev E 1961 Periodically correlated random sequences Sov. Math. 2 385-8

[32] Gray H L and Zhang N F 1988 On a class of nonstationary processes J. Time Ser. Anal. 9 133-54

[33] Hannan E 1965 Group representations and applied probability Supplementary Review Series in Applied Probability vol 3 ed J Gani (London: Methuen \& Co)

[34] Huang Y, Johansen A, Lee M W, Saleur H and Sornette D 2000 Artifactual log-periodicity in finite-size data: relevance for earthquake aftershocks J. Geophys. Res. 105 25451-71

[35] Lamperti J 1962 Semi-stable stochastic processes Trans. Am. Math. Soc. 104 62-78

[36] Lim S C and Muniandy S V 2003 Generalized Ornstein-Uhlenbeck processes and associated self-similar processes J. Phys. A: Math. Gen. 36 3961-82

[37] Loève M 1962 Probability Theory (Princeton, NJ: Von Nostrand-Reinhold)

[38] Mallat S 1999 A Wavelet Tour of Signal Processing (New York: Academic)

[39] Mandelbrot B 1982 The Fractal Geometry of Nature (San Francisco, CA: Freeman)

[40] Mandelbrot B 1999 A multifractal walk down Wall Street Sci. Am. 280 70-3

[41] Mandelbrot B and Van Ness J 1968 Fractional Brownian motions, fractional noises and applications SIAM Rev. $10422-37$ 
[42] Noret E and Guglielmi M 1999 Modélisation et synthèse d'une classe de signaux auto-similaires et à mémoire longue Proc. Conf. Delft (NL): Fractals in Engineering (INRIA) pp 301-15

[43] Nottale L 1993 Fractal Space-Time and Microphysics (Singapore: World Scientific)

[44] Nuzman C and Poor V 2000 Linear estimation of self-similar processes via Lamperti's transformation J. Appl. Probab. 37 429-52

[45] Park K and Willinger W (ed) 2000 Self-Similar Network Traffic and Performance Evaluation (New York: Wiley)

[46] Perrin O and Senoussi R 1999 Reducing non-stationary stochastic processes to stationarity by a time deformation Stat. Probab. Lett. 43 393-7

[47] Saleur H and Sornette D 1996 Complex exponents and log-periodic corrections in frustrated systems J. Phys. I France 6 327-55

[48] Samorodnitsky G and Taqqu M 1994 Stable non-Gaussian Random Processes: Stochastic Models with Infinite Variance (London: Chapman and Hall)

[49] Sampson P and Guttorp P 1992 Nonparametric estimation of nonstationary spatial covariance structure $J$. Am. Stat. Ass. 87 108-19

[50] Silverman R 1957 Locally stationary random processes IRE Trans. Inform. Theory 3 182-7

[51] Sornette D 1998 Discrete scale invariance and complex dimensions Phys. Rep. 297 239-70

[52] Sornette D 2000 Critical Phenomena in Natural Sciences (New York: Springer)

[53] Vervaat W 1987 Properties of general self-similar processes Bull. Int. Stat. Inst. 52 199-216

[54] Vidács A and Virtamo J 1999 ML estimation of the parameters of fBm traffic with geometrical sampling IFIP TC6, Int. Conf. on Broadband Communications'99 (Hong Kong, November 1999)

[55] Wood A T A and Chan G 1994 Simulation of stationary process in [01] ${ }^{d}$ J. Comput. Graph. Stat. 3 409-32

[56] Wornell G 1996 Signal Processing with Fractals: A Wavelet-Based Approach (Englewood Cliffs, NJ: PrenticeHall)

[57] Yazici B and Kashyap R L 1997 A class of second-order stationary self-similar processes for $1 / f$ phenomena IEEE Trans. Signal Process. 45 396-410 